\title{
Spontaneous cervical subcutaneous and mediastinal emphysema secondary to occult sigmoid diverticulitis
}

\author{
T. Hur*, Y. Chen**, G.H.F. Shu', J-M. Chang', K-C. Cheng
}

Spontaneous cervical subcutaneous and mediastinal emphysema secondary to occult sigmoid diverticulitis. T. Hur, Y. Chen, G.H.F. Shu, J-M. Chang, K-C. Cheng. (OERS Journals Ltd 1995.

ABSTRACT: We present a case of spontaneous mediastinal and subcutaneous cervical emphysema due to perforation of an occult sigmoid diverticulitis.

Mediastinal emphysema should alert the physician to the possibility of retroperitoneal gastrointestinal perforation, even in patients without signs of distinct peritoneal irritation.

Eur Respir J., 1995, 8, 2188-2190.

Spontaneous supraclavicular emphysema is relatively common as a sequela of pulmonary interstitial emphysema and pneumomediastinum. It is often the result of alveolar wall rupture secondary to high intra-alveolar pressure caused by artificial ventilation, coughing, and straining [1]. However, it is quite rarely associated with intestinal perforation, particularly in cases with no history of instrumental manipulation of the hollow abdominal viscera [2].

\section{Case report}

A 67 year old woman was referred to our emergency service because of sudden onset of marked neck swelling for $2 \mathrm{~h}$ without concomitant dyspnoea. This was preceded by a slight lower back soreness for about 1 week. She had visited our orthopaedic clinic 3 days before, where degenerative disc disease with compression fracture of L4-5 was disclosed after radiographic imaging of the spine. The patient had previously been in good health, with the exception of diabetes mellitus, which had not been satisfactorily controlled.

On physical examination, the patient appeared slightly ill-looking with a temperature of $36.4 \%$ blood pressure of $160 / 90 \mathrm{mmHg}$, pulse rate of 84 beats $\cdot \mathrm{min}^{-1}$, and respiratory rate of 16 breaths $\cdot \mathrm{min}^{-1}$. The patient's neck demonstrated swelling and crepitation, but no significant lymphadenopathy was found. Chest examination was normal. The abdomen was slightly distended and vaguely tender over the lower region, without signs of peritoneal irritation. Peristalsis remained active and bowel function was normal. However, knocking pain over both loins seemed equivocal. A chest roentgenogram demonstrated an unexpected pneumomediastinum, but no free air was apparent in the abdomen (fig. 1).
Depts of *Pulmonary and Critical Care. **General Surgery and +Diagnostic Radiology, Chi-Mei Foundation Hospital, Tainan, Taiwan, Republic of China

Correspondence: T. Hur, Dept of Medicine, Chi Mei Foundation Hospital, 901 Chung Hwa Road, Yung Kang City, Tainan, 71010 Taiwan, Republic of China

Keywords: Pneumomediastinum, pneumoretroperitoneum, sigmoid colon

Received: March 1 1995; Accepted after revision July 31995

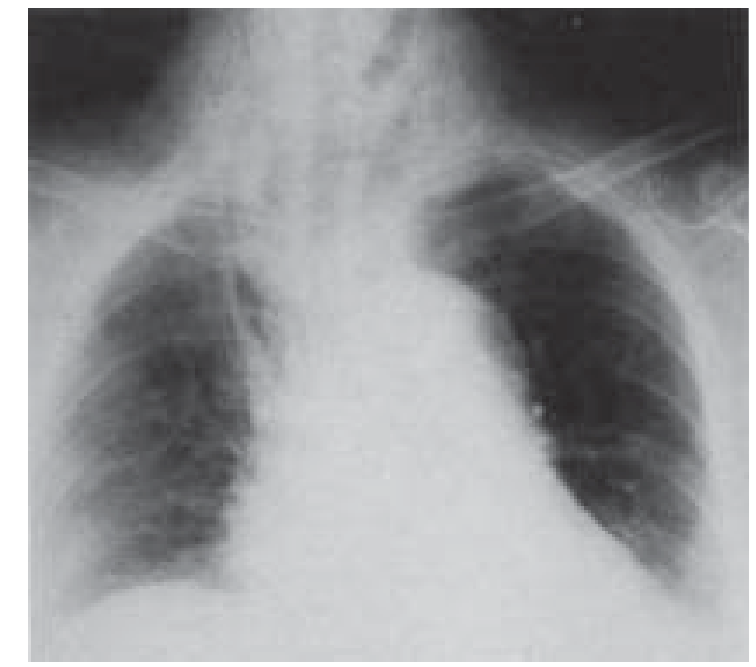

Fig. 1. - Chest roentgenogram showing subcutaneous emphysema and pneumomediastinum. No air was demonstrated in the peritoneal cavity.

Complete blood count (CBC) showed a haemoglobin level of $141 \mathrm{~g} \cdot \mathrm{L}^{-1}$, a white blood cell count (WBC) of $20.6 \times 10^{9}$ cells $\cdot \mathrm{L}^{-1}$ and a platelet count of $218 \times 10^{9} \cdot \mathrm{L}^{-1}$. The faeces were positive $(+++)$ for occult blood. Blood sugar level was $3.6 \mathrm{~g} \cdot \mathrm{L}^{-1}$ (normal range $0.7-1.1 \mathrm{~g} \cdot \mathrm{L}^{-1}$ ), and alkaline phosphatase was $147 \mathrm{U} \cdot \mathrm{L}^{-1}$ (normal range 25$\left.80 \mathrm{U} \cdot \mathrm{L}^{-1}\right)$.

Fibreoptic bronchoscopic examination produced no evidence of pulmonary disruption. Gastroduodenoscopy was also performed, which revealed only multiple small gastric ulcers at the antrum, primarily along the side of greater curvature, and still with no sign of perforation. After 5 days of intravenous antibiotics and oral hypoglycaemic treatment, the WBC and glucose level returned to normal ranges. 
A computed tomographic (CT) examination of the chest showed some irregular small pockets of air collection in the fascial planes of the mediastinum and the preaortic region. Free air was also noted in the retroperitoneum, suggesting some communication between the chest and the retroperitoneum. The plain abdominal radiograph confirmed the presence of retroperitoneal air outlining the kidneys and psoas musculature. A CT scan of the abdomen showed gas from the posterior mediastinum to the gastrophrenic, gastrosplenic, gastrocolic, and splenorenal ligaments. Involvement of the mesentery and bilateral anterior and posterior pararenal spaces to the pelvic extraperitoneal space was also noted (fig. 2a). CT scan of the pelvis after lower gastrointestinal (LGI) series eventually revealed a pelvic abscess above the sigmoid colon (fig. 2b). a finding that the LGI series itself could not clearly demonstrate, apart from three diverticula nearby. Gynaecological examination did not reveal anything abnormal.

Peritoneal signs appeared on the 12th day after admission. However, the patient still remained afebrile. An urgent exploratory laparotomy was undertaken, under the suspicion of sigmoid diverticulitis rupture with abscess formation. During the operation, a perforation over the posterior wall of the sigmoid colon was found, surrounded by a large walled-off abscess. Segmental resection of the sigmoid colon and end-colostomy (Hartmann's procedure) were performed. Grossly, the resected bowel measured $12 \mathrm{~cm}$ in length and $7 \mathrm{~cm}$ in circumference. The

a)

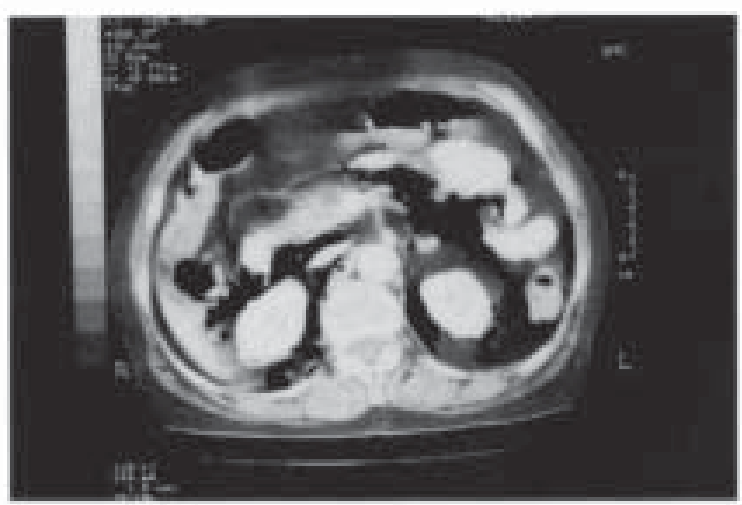

b)

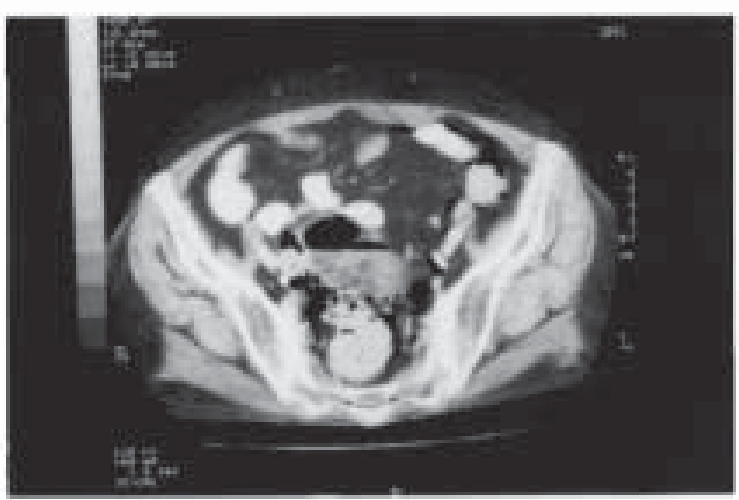

Fig. 2. - a) Computed tomographic (CT) scan of the abdomen showing gas surrounding bilateral anterior and posterior pararenal spaces. b) CT scan of the pelvis showing a pelvic abscess above the sigmoid colon. hole measured $0.7 \times 0.6 \mathrm{~cm}$, and its surrounding mucosa was erythematous and thinned.

Histologically, severe inflammatory change with a mixed population of leucocytes, oedema, and fresh haemorrhage at the edges of the rupture site were observed, with no sign of malignancy. Culture of the pus revealed mixed infection both of aerobic and anaerobic microorganisms, including Pseudomonas aeruginosa, Escherichia coli, Gr. D enterococcus (Streptococcus faecalis), and B-haemolytic Gr. G Streptococcus. The patient's subcutaneous emphysema and pneumomediastinum disappeared quickly after the operation. She made a very satisfactory recovery and was discharged with a colostomy after 4 weeks. Five months later, the patient was still healthy.

\section{Discussion}

Subcutaneous emphysema of the neck may be found in bronchopulmonary disruption, gas forming infections, and a tear or perforation in the gastrointestinal tract. In the literature, we found only a few reports on subcutaneous emphysema of gastrointestinal origin, particularly for those cases in which the gas collection is located in the supraclavicular area [3-5]. In fact the presentation of emphysema is determined largely by the anatomical location of the primary perforation. Generally, emphysema of the anterior abdominal wall is associated with perforation of the small intestine, appendix and colon; emphysema of the scrotum, perineum, or thigh is usually associated with tears in the anorectal area or colon. Supraclavicular emphysema is most commonly associated with a perforated gastric ulcer.

Subcutaneous emphysema of gastrointestinal origin usually occurs after surgical procedures as the direct result of the operation itself or following leakage of suture lines, fistula formation, or infections. Often causes include Boehaave's syndrome (spontaneous perforation of the oesophagus), perforated peptic ulcers, traumatic perforation, and appendicitis. In the three cases that we found in the literature to be associated with colonic diverticulitis, subcutaneous emphysema occurred after surgery and was located mainly in the lower abdomen, thigh and perineum [3]. Therefore, the current case, with spontaneous neck and mediastinal emphysema resulting from an occult perforation of the sigmoid diverticulitis, is quite original.

The anatomical site of perforation largely determines the route of escape of the gas to the subcutaneous position. In addition, the direction of gas diffusion usually follows the least resistance, loose areolar fascial structures. In this case the perforation was located in the posterior wall of the sigmoid colon, and then penetrated directly into the retroperitoneal space. The persistent peristalsis of the colon may have contributed to large pressure gradient between the bowel lumen and the retroperitoneum, which caused the intestinal contents and gas to be pushed into the retroperitoneal space. The escaped gas may continue diffusing superiorly through the paravertebral retroperitoneal tissues and diaphragmatic hiati into the mediastinum, and then into the neck and facial areas. 
Invasive instrumental manipulation, such as endoscooic sphincterotomy, colonscopic polypectomy, and laparoscopy may induce the perforation of the hollow abdominal viscera, which commonly presents as haemorrage and peritonitis. However, there were also reports of subcutaneous emphysema, an initial presentation that provides, important evidence of the perforation $[4,5]$.

The diagnosis of retroperitoneal perforation is usually impeded by the lack of signs of peritoneal irritation. In one study, investigating perforative carcinoma of the colon and rectum, the median duration of abdominal symptoms was reported to be 1.7 months in patients with retroperitoneal perforation and abscess formation, compared with 1 week in intraperitoneal-free perforation with peritonitis [6]. A timely roentgenographic examination of the abdomen is invaluable in determining the presence of retroperitoneal gas, as in this case.

\section{References}

1. Maunder RJ, Pierson DJ, Hudson LD. Subcutaneous and mediastinal emphysema: pathophysiology, diagnosis and management. Arch Intern Med 1984; 144: 1447-1453.

2. Humphreys F, Hewetson KA, Dellipiani AW. Massive subcutaneous emphysema following colonscopy. Endoscopy 1984; 16: 160-161.

3. Oetting HK, Kramer NE, Branch WE. Subcutaneous emphysema of gastrointestinal origin. Am J Med 1955; 19: 872-886.

4. Bakker J, Kerson FV. Spruyt JB. Pneumopericardium and pneumomediastinum after polypectomy. Endoscopy 1991; 23: 46-47.

5. Walker HC, Nivatvongs S, Ansel HJ, Gedgaudas E. Massive extraperitoneal air in a 71 year old woman: occurence during a radiological study. J Am Med Assoc 1982; 248: 1375-1376.

6. Welch JP, Donaldson GA. Perforative carcinoma of the colon and rectum. Ann Surg 1974; 180: 734-740. 